\title{
Aprendiencia en la docencia y construcción de conocimiento biopedagógico
}

\section{Learning Process in Teaching and Bio-pedagogical Knowledge Building}

\author{
Luz Emilia Flores Davis' \\ División de Educación Básica \\ Centro de Investigación y Docencia en Educación \\ Universidad Nacional \\ Heredia, Costa Rica \\ luzdeflores@gmail.com
}

Recibido 05 de mayo de 2012 • Corregido 26 de junio de 2012 • Aceptado 28 de junio de 2012

Resumen. La biopedagogía se edifica en la praxis, es decir, en la interrelación de reflexión y en la acción innovadora, las cuales se amalgaman en la construcción de sentidos y generan conocimiento. Surge, entonces, la pregunta: ¿cómo se aprehende la docencia, cómo se logra renovar la práctica desde la acción-reflexión-acción de manera recursiva y en la vida misma? Una alternativa para la búsqueda de respuestas es la sistematización de experiencias, la cual constituye una modalidad de investigación cualitativa. Promueve la transformación de una práctica, a partir de la construcción de conocimiento desde abordajes holísticos de la complejidad del hecho educativo. La sistematización de experiencias biopedagógicas asume la autoorganización, el gozo, la incertidumbre y la pasión; respeta la libertad, la autonomía y genera espacios relacionales que promueven la expresión de procesos creativos en la aprendiencia docente.

Palabras claves. Aprendiencia, biopedagogía, sistematización, docencia.

Abstract. Bio-pedagogy is built on praxis, i.e. the interrelationship between reflection and innovative action where these two merge in the construction of senses to generate knowledge. Then, the following question arises: How is teaching understood? How can practice be renovated from the action-reflection-action in a recurring manner and in life itself? A way to search for those answers is the systematization of experiences -a modality of qualitative research. It promotes the transformation of a common practice, based on knowledge building by holistic approaches to the educational process complexity. The systematization of bio-pedagogical experiences involves self-organization, joy, uncertainty and passion; it respects freedom and autonomy, and generates relational spaces, which promote creative processes in learning.

Keywords. Learning process, bio-pedagogy, systematization, teaching.

Doctora en Educación, Máster en Educación Superior, Catedrática universitaria. Ha ocupado los cargos de Directora de Docencia, Directora de Desarrollo Profesional y Cooperación Universitaria, Presidenta de la Junta de Becas y Coordinadora de la Comisión Técnica de Admisión de la Universidad Nacional de Costa Rica. Actualmente, se desempeña como investigadora, extensionista y docente en la División de Educación Básica del CIDE y docente en la Maestría en Formación Dancística del CIDEA. 
URL: http://www.una.ac.cr/educare

\section{Introducción}

La biopedagogía se edifica en la praxis, es decir, en la interrelación de reflexión y en la acción transformadora, las cuales se amalgaman en la construcción de sentidos y generan conocimiento. Mis interrogantes fundamentales en este artículo se refieren a cómo se aprehende la docencia, cómo se logra transformar la práctica desde la acción-reflexión-acción de manera recursiva y en la vida misma.

Hemos aceptado que es necesario recurrir a la investigación para ese propósito, pero me pregunto, ¿qué características responden a la investigación en la biopedagogía en el marco del paradigma emergente?; ¿existen estrategias alternativas para conocer las realidades e innovarlas?

Para contextualizar mis preguntas, es necesario compartir una inquietud que me acompaña desde hace bastantes años, y que se originó cuando fungía como tutora de trabajos finales de graduación en la modalidad de tesis. Me pregunto, desde entonces, por qué parece que muchas estudiantes y muchos estudiantes y docentes que realizan sus tesis no las disfrutan, por qué no les apasiona lo que investigan, por qué luego de aprobar su tesis no continúan investigando en su labor docente; por qué quienes mayoritariamente generan conocimiento para el ámbito pedagógico son especialistas de otras disciplinas y no las pedagogas y los pedagogos.

Asumo, entonces, la afirmación de Freire (2003): “La realidad no es así, la realidad está así. Y está así no porque ella quiera. Ninguna realidad es dueña de sí misma" (p. 63).

\section{Biopedagogía}

La biopedagogía nace en el marco del nuevo paradigma, caracterizado por su perspectiva holística y ecológica. El paradigma emergente tiene una visión comprometida con la vida y considera que existe una red de conexiones e interacciones de complementariedades entre todos los seres vivos.

En la biopedagogía se entiende que aprendizaje y vida son un solo proceso, que se aprende para vivir y el aprender es una condición fundamental del estar vivo o viva (...) La biopedagogía del aprendizaje posee un amplio sentido de compromiso ecológico y social, y se estructura desde el cambio y potenciación de quien aprende. (L. E. Flores, G. Flores, Jiménez, Madrigal, y Perearnau, 2009, p. 21)

La biopedagogía conlleva la capacidad de autoorganizarse para aprender con placer en un proceso dinámico y recursivo, es decir, un proceso "cuyos productos son necesarios para la propia producción del proceso" (Morin,Roger y Motta, 2003, p. 40). 
Humberto Maturana aporta a la biopedagogía el concepto de autopoiesis, cuyo significado se refiere a la capacidad de los seres vivos de automantenerse a sí mismos. Lo concibe desde la teoría de la biología del amor, fundamentada en asumir al otro como un legítimo otro (Maturana, 1999).

El espacio educacional, como un espacio de coexistencia en la biología del amor, debe ser vivido en el placer y alegría de ver, tocar, oír, oler, y reflexionar, que nos torna capaces de ver, oír, oler y tocar todo lo que llega a ser accesible para nosotros cuando tenemos libertad para mirar, y miramos simultáneamente al contexto y a la peculiaridad de la situación en la cual estamos en cualquier instante, y hacemos esto abiertos a relacionar situación y contexto sin temor. (Maturana, 1999, p. 67)

Esta manera de entender la educación conlleva procesos de mediación biopedagógica que respetan la libertad de quienes aprenden, y promueve la autonomía. La biopedagogía asume como una de sus premisas la aprendiencia desde la complejidad y la diversidad. Acoge la incertidumbre, la cognición estética y promueve el cuidado. "El cuidado sirve de crítica a nuestra civilización agonizante y también de principio inspirador de un nuevo paradigma de convivencia" (Boff, 2001, p. 12).

\section{Aprendiencia}

El vocablo "aprendizaje" debe dejar lugar a un nuevo vocablo "aprendiencia", que expresa mejor, por su propia forma, este estado de "estar-en-proceso-de-aprender", esta función del acto de aprender que construye y se construye, y su categoría de acto existencial que caracteriza efectivamente el acto de aprender, indisociable a la dinámica de los seres vivos. (Trocmé-Fabre, 1997, citado por Assmann, 2002, p. 15)

En ese marco se inscribe el concepto de aprendientes tanto aplicado a personas, comunidades, sociedades u organizaciones, y responde a un cambio de enfoque pedagógico.

La noción de "alumno" que conlleva una relación de subordinación y acato al profesor, cambió al vocablo de "estudiante" en los enfoques participativos, dados los mayores niveles de emancipación que pretenden en la relación educativa; ahora bien, en el marco de la biopedagogía, este concepto evoluciona a "aprendientes".

En los enfoques tradicionales hablar de profesor y de alumno es coherente con sus principios de relaciones verticales, donde se asume la docencia como sitio de transmisión de 
URL: http://www.una.ac.cr/educare

contenidos programáticos, y quien "sabe" informa a quien "no sabe". El avance hacia mayores niveles de participación en los procesos educativos cambió el concepto de profesor a facilitador y como ya se dijo de alumno a estudiante.

En la biopedagogía se da un giro no solo hacia relaciones más horizontales, sino más bien a la valoración de las interacciones sociales, y se propone el término de mediador o mediadora, así como de aprendientes; no obstante, conforme se avanza en esta búsqueda de alternativas más coherentes con los principios del paradigma emergente, estamos caminando hacia la transformación de las aulas y demás espacios pedagógicos, en comunidades aprendientes, donde las relaciones conllevan con-vivir en comunión, con un auténtico diálogo, y en un compartir en solidaridad y respeto.

La aprendiencia es propia de la biopedagogía, pues estar vivo significa estar aprendiendo.

\section{Una estrategia de aprendiencia: Sistematización de experiencia}

Es distinto sistematizar informaciones a sistematizar experiencias. La sistematización de la información permite estructurar el conocimiento, según una lógica interna de la disciplina o asignatura, pero cuando nos referimos a la sistematización de experiencias estamos hablando de la transformación de una práctica a partir de la construcción de conocimiento, y reconociendo la complejidad del hecho educativo.

La sistematización y la investigación evaluativa (...) son modalidades de investigación de y en [sic] la práctica, puentes para producir conocimiento y reflexión crítica sobre la misma [sic]. Son dos instancias que permiten mediar en la tensión teoría-practica y posibilitan una intervención reflexiva y dinámica, acorde con los acelerados cambios que presenta la sociedad contemporánea y los desafíos y retos que tiene la profesión para intervenir en dicha complejidad. (Pérez, 2009, p. 48)

La sistematización implica revivir la experiencia y generar conocimiento; en ello concuerdan diversos autores tales como Jara (1994), Martinic y Walter (1999), Quiroz y Morgan (1998), Pierola (1987) y Sánchez (1986), citados por Pérez (2009).

"La sistematización de experiencias es considerada como una modalidad de investigación cualitativa, orientada a la producción de sentidos mediante el análisis crítico y autocrítico (...)". (Sánchez, 2010, p. 6)

En la sistematización de experiencias biopedagógicas se realiza una interpretación crítica que permite comprender por qué se dan ciertas situaciones, por qué la mirada está enfocada en 
determinados aspectos de la realidad y no en otros, qué se quiere mantener en esa realidad y qué se desea que cambie; qué teoría emana de la práctica y cómo se pueden compartir los aprendizajes.

\section{Una vivencia representativa de aprendiencia docente}

En el programa de Maestría en Danza con énfasis en Formación Dancística, de la Escuela de Danza, del CIDEA, en la Universidad Nacional de Costa Rica, se ha optado por la sistematización de experiencia para que las aprendientes y los aprendientes integren los conocimientos construidos durante el desarrollo de la Maestría y de su vida plena.

Esa vivencia se realiza durante dos cursos que entrelazan Danza y Educación. En ellos los sujetos aprendientes llevan a cabo una praxis de formación dancística, a lo menos dos veces por semana, durante dieciocho semanas, con un grupo de participantes del nivel principiante, intermedio o avanzado, y sistematizan su experiencia. Concluida cada sesión, o cada semana, escriben una crónica donde registran, mediante una descripción, la clase llevada a cabo, y redactan un comentario o reflexión personal. Se les invita a apuntar preguntas que surgen de sus introspecciones, referidas a cualquier aspecto de la vivencia docente. Paralelamente a la redacción de las crónicas, toman en video muestras de sus clases, al inicio, durante y al final del proceso formativo.

Para la elaboración del Informe de sistematización se utiliza un itinerario con un amplio grado de flexibilidad que sirve de orientación general. No obstante, cada aprendiente redacta el informe desde su visión, a su manera, y profundiza en aquello que considere de mayor relevancia.

Ese itinerario está constituido por una introducción, donde se presenta tanto el contenido del documento, como su autora o autor, texto que nace de una reflexión autobiográfica; se justifica el proceso llevado a cabo; se hace referencia a los antecedentes; y se explicitan los propósitos o intencionalidades de la sistematización. Posteriormente, se comparte el marco conceptual del que participa el autor o la autora del informe. Luego se aborda, a manera de marco metodológico, la contextualización de la experiencia llevada a cabo, las características de las participantes y los participantes, el programa del curso con su respectiva estructura de clase y los planeamientos elaborados para las lecciones. Continúa el informe con un marco interpretativo que reúne las crónicas y las preguntas que se generaron, así como una interpretación crítica general. Como último apartado, se plantean conclusiones o revelaciones con los aprendizajes relevantes, una o varias propuestas para el mejoramiento de la formación dancística y recomendaciones dirigidas a diversas instancias, según sea el caso. El informe finaliza con las referencias utilizadas y anexos, si se requiere.

Como se desprende del planteamiento teórico que precede a la propuesta de itinerario, los apartados del informe no se elaboran en secuencia lineal, sino se van construyendo durante la sistematización. La riqueza de los informes radica en su originalidad y autenticidad. Son la expresión de procesos creativos en la aprendiencia docente. 
URL: http://www.una.ac.cr/educare

\section{Conclusiones}

La docencia se aprehende y se transforma mediante la toma de conciencia de aprendiencia y conlleva construcción de conocimiento biopedagógico de manera dinámica. La sistematización de experiencias es una estrategia alternativa para conocer las realidades e innovarlas, es una opción de investigación cualitativa que responde a los planteamientos de la biopedagogía en la búsqueda de abordajes holísticos de la complejidad educativa. Así, la sistematización de experiencias respeta la libertad, la expresión, la autonomía y genera espacios relacionales que promueven la creación. Asume la autoorganización en la acción, la reflexión, el gozo, la incertidumbre y la pasión.

A continuación y en la búsqueda de nuevas posibilidades inspiradoras para la aprendiencia docente, transcribo algunos extractos de textos de los Informes de Sistematización de aprendientes de los cursos que he tenido el privilegio de compartir desde el año 2004 hasta la fecha, con las cinco generaciones de Maestría en Danza².

"Es la vida una complejidad inmensa y a la vez tan simple, conlleva matices profundos de una realidad existente muchas veces de experiencias dolorosas, amenas, retadoras, contradictorias, divertidas, de encuentros, despedidas, de búsquedas y aprendizajes." A. L.

"Subirse al escenario para danzar es escribir poesía en el espacio, gozar de soledad con el cuerpo, es regresar a uno mismo, significa que estamos sembrándonos a nosotros mismos para ser entes vivos con ganas de ser parte de la Tierra y de la Vida." I. L.

"El cuerpo, la mente y el alma danzan todo el tiempo, con el más mínimo e imperceptible movimiento. Se fusionan, a veces cantan, ríen, Iloran, gritan, murmuran, alaban, agradecen, ofrecen, seducen, protestan..., aún en la inmovilidad del cuerpo físico existe el diálogo entre ellos. Esto es el lenguaje del cuerpo." S. 0.

“El hecho de asumir la práctica docente como un proceso de investigación para las propias acciones e interacciones con los estudiantes fortaleció enormemente el aspecto creativo de dicha práctica tanto para mí como para los estudiantes. Lo importante de la investigación en el campo pedagógico es que permite analizar y reflexionar acerca de la propia labor formativa y compartir con otros las experiencias." I. A.

2 Se utilizan iniciales de los nombres de las aprendientes y los aprendientes que expresaron sus opiniones, con el propósito de proteger su identidad. 
“Esta experiencia docente es una prueba más, de que constantemente estamos aprendiendo y retroalimentándonos de conocimientos, en la misma práctica de enseñar..." P. B.

"En el espacio vacío voy escribiendo con la voz de mi corporalidad, y en este recorrido presente, en el que está el futuro impredecible, voy vivenciando cada instante... No sé por ahora el punto de llegada, aunque intuitivamente pueda imaginar hacia dónde me dirijo." E. L.

“Este escrito permite viajar entre historia, teorías y métodos, para comprender de qué manera se construye un aprendizaje, y cómo, todo lo que nos rodea en nuestro entorno influye y cala en el desarrollo humano." M. $\mathbf{O}$.

“El proceso de sistematización genera la autorreflexión, ya que en la realidad se da un ciclo constante de mejora. Un aspecto importante a destacar es que la misma autorreflexión y análisis generó una revisión de los objetivos propuestos, dando lugar a la inclusión de nuevos temas que no se habían contemplado y que han surgido precisamente por la flexibilidad de las lecciones." H. M.

"El "error", que siempre está presente en todos nuestros aciertos, es fuente de inspiración para nuevos descubrimientos y la diversidad es considerada como nuestro gran capital, que se puede utilizar y madurar con el trabajo diario." R. S.

"Cada clase fue única, el trabajo que se realizó dentro de ella fue un trabajo en equipo, lo que proponía para la clase no era algo definitivo, estaba sujeto a cambios y a sugerencias expuestas por las estudiantes, lo que implica que todo planeamiento debe ser flexible y adaptarse a las circunstancias de la lección y a las necesidades de las estudiantes." C. S.

"Vuelvo entonces a analizar a este grupo de personas con el que he decidido trabajar (...) Me cautivan sus miradas inquietas llenas de entusiasmo y vitalidad, de preguntas, de búsqueda, y reuniendo todo esto me llena de placer el saber que entrego y reciben, y recibo yo todo aquello que ellos me entregan, a su vez. Va y viene una energía como olas del mar. Va y viene una pasión que no se calla, que no se limita simplemente a estar ahí, sino que vibra en todo su esplendor y crece." M. V. 
URL: http://www.una.ac.cr/educare

"Con el transcurrir de la práctica docente, la retroalimentación de experiencias entre las alumnas y yo, se convirtió en uno de los ejes centrales de todo el proceso. Hay que entender que la enseñanza y el aprendizaje, deben tener una correlación, en la que se intercambian los resultados de las vivencias del estudiante y del maestro." L. C.

“El maestro (...) debe utilizar como herramienta imprescindible la creatividad y convertirse en un creador, en un artista, pues el éxito de su labor estará siempre supeditado a infinidad de variables, la mayoría impredecibles." O.C.

"Este difícil proceso (la sistematización de experiencia), nuevo para muchos de nosotros que tenemos años de impartir clases, (...) se convirtió en tiempo de reflexiones, tiempo de autoevaluación, tiempo de crecer, tiempo de confusión, tiempo de continuar adelante, tiempo para decidir ser mejor persona, tiempo para decidir ser mejor profesional de la danza." R. B.

“El escribir las crónicas después de cada clase me ha enriquecido (...), estas me han ayudado a observar detenidamente a cada una de las alumnas, sus expresiones, sus logros, sus temores y hasta sus intereses, (...) he logrado sentir y ver el progreso de cada una de las niñas en un ambiente, fresco, cálido, en donde reina la autodisciplina y la felicidad por aprender." R. C.

¿Qué aprendí? (...) Aprendí que la belleza, la humildad y la mejoría está precisamente en la simplicidad y que todo lo que se trabaja a conciencia con mucho optimismo por el simple hecho de aprender sin vanidad alguna, se convierte en talento, como si fuéramos el mejor de los pianistas, o la más etérea y delicada bailarina." M. L.

“Después de completar esta sistematización, logro alcanzar una nueva percepción de mi forma de enseñanza, una visión más analítica y crítica, que me ha permitido tener la capacidad de poder observar en mi docencia, al antes y después de un proceso de aprendizaje significativo..." M. S.

"Pero mi escuela -académicamente hablando- fue la vivencia que fui y sigo adquiriendo en mi experiencia profesional. Así pasé mucho tiempo, muchos años, buscando; pero, ¿acaso existe un orden correcto para expresarse?, ¿existe algún secreto o varita mágica para ser ungido con la justa inspiración, que dará la justa expresividad?" V. C. 


\section{Referencias}

Assmann, H. (2002). Placer y ternura en la educación. Hacia una sociedad aprendiente Madrid: Narcea.

Boff, L. (2001). Cuidar la Tierra. Hacia una ética universal. México: Dabar.

Flores, L.E., Flores, G., Jiménez, R., Madrigal. J. C. y Perearnau, M. A. (2009). Comunidad aprendiente. San José, Costa Rica: Artemusa.

Freire, P. (2003). El grito manso. Buenos Aires: Siglo XXI.

Maturana, H. (1999). Transformación en la convivencia. Chile: Dolmen Ediciones.

Morin, E., Roger, E. y Motta, R. D. (2003). Educar en la era planetaria. España: Gedisa.

Pérez, A. (agosto, 2009). Re-pensar la sistematización y la investigación evaluativa en la intervención del trabajo social, como pilares para la producción de conocimiento. Palabra, 10, 42-56.Recuperado de http://aula.tcomunica.org/wp-content/uploads/2009/03/ art\%C3\%ADculo-Alexander-Pérez-Álvarez.pdf

Sánchez, A. (febrero-mayo, 2010). El artículo sistematización de experiencias: Construcción de sentido desde una perspectiva crítica. Revista Virtual Universidad Católica del Norte, 29, 1-7. Recuperado de http://redalyc.uaemex.mx/redalyc/pdf/1942/194214466001.pdf 